\title{
Excitation Spectrum of the Néel Ensemble of Antiferromagnetic Nanoparticles as Revealed in Mössbauer Spectroscopy
}

\author{
Mikhail A. Chuev \\ Institute of Physics and Technology, Russian Academy of Sciences, Nakhimovskii pr. 36-1, Moscow 117218, Russia \\ Correspondence should be addressed to Mikhail A. Chuev; chuev@ftian.ru
}

Received 13 November 2016; Accepted 8 March 2017; Published 11 June 2017

Academic Editor: Jan A. Jung

Copyright ( 2017 Mikhail A. Chuev. This is an open access article distributed under the Creative Commons Attribution License, which permits unrestricted use, distribution, and reproduction in any medium, provided the original work is properly cited.

\begin{abstract}
The excitation spectrum of the Néel ensemble of antiferromagnetic nanoparticles with uncompensated magnetic moment is deduced in the two-sublattice approximation following the exact solution of equations of motion for magnetizations of sublattices. This excitation spectrum represents four excitation branches corresponding to the normal modes of self-consistent regular precession of magnetizations of sublattices and the continuous spectrum of nutations of magnetizations accompanying these normal modes. Nontrivial shape of the excitation spectrum as a function of the value of uncompensated magnetic moment corresponds completely to the quantum-mechanical calculations earlier performed. This approach allows one to describe also Mössbauer absorption spectra of slowly relaxing antiferromagnetic and ferrimagnetic nanoparticles and, in particular, to give a phenomenological interpretation of macroscopic quantum effects observed earlier in experimental absorption spectra and described within the quantum-mechanical representation.
\end{abstract}

\section{Introduction}

A wide application of materials containing nanosized antiferromagnetic particles in different branches of nanotechnology is primarily due to a number of specific structural, magnetic, and thermodynamic properties of these materials found within long-term fundamental studies. However, these real materials are still characterized by different experimental techniques on the basis of phenomenological Néel approach describing a superposition of antiferromagnetism and superparamagnetism of uncompensated magnetic moments on two magnetic sublattices [1,2]. Practical models for analyzing experimental data taken on antiferromagnetic nanoparticles are based mainly on the representation of uncompensated magnetic moment and a rather simplified treatment of antiferromagnetism in terms of linear magnetic susceptibility introduced also by Néel $[3,4]$. However, the ground state for antiferromagnetic nanoparticles should be much more complicated as compared to that for a bulk sample, which is evidenced from the atomic-scale magnetic modeling $[5,6]$ that in its turn is hardly possible to be used for analyzing experimental data in practice due to computational expenses followed by an uncontrollable accuracy of calculations.
Meanwhile, a quantum-mechanical model for describing thermodynamic properties of an ensemble of ideal (compensated) antiferromagnetic nanoparticles is recently developed $[7,8]$. This model clarifies principally the difference in thermodynamic behavior of ferromagnetic and antiferromagnetic particles revealed in spectroscopic measurements without the assistance of uncompensated magnetic moment and describes qualitatively macroscopic quantum effects earlier observed repeatedly in experimental Mössbauer absorption spectra of antiferromagnetic and even ferrimagnetic nanoparticles [9-13]. It was also shown that taking the uncompensated spin in the account does not change the qualitative pattern of these effects but is reduced to small numerical corrections of the shape of the absorption spectrum of the ensemble of antiferromagnetic particles [14]. Note that the earlier studies of macroscopic quantum phenomena in small antiferromagnetic particles have also explored the same Néel idea of the uncompensated magnetic moment, but again within a simplified treatment of the ground state and the lowest energy levels $[15,16]$.

The quantum-mechanical model $[7,8,14]$ can be easily realized in numerical calculations and efficiently used for 
analyzing experimental data, in particular, for analyzing a large array of Mössbauer spectra taken at different temperatures [17]. However, for solving the last task in a complete manner one should take into consideration relaxation processes that is the corresponding model of magnetic dynamics to be developed. On the other hand, the macroscopic quantum effects observed in the Mössbauer spectra of antiferromagnetic nanoparticles are treated within the quantummechanical model $[8,14]$ only in terms of wave functions and mean values of macrospins of sublattices for different energy levels with no phenomenological explanation. The latter can be found only in the macroscopic limit. The corresponding continuum model of the magnetic dynamics of an ensemble of ideal antiferromagnetic nanoparticles in the two-sublattice approximation is recently proposed in solving the equations of motion for magnetizations of sublattices [18]. This model has demonstrated the nontrivial character of the excitation spectrum of particles in the form of four excitation branches corresponding to four normal modes of self-consistent uniform precession of vectors of magnetizations of sublattices around the easy axis. Two of these modes are known well from the classical theory of antiferromagnetic resonance in the zero external magnetic field $[19,20]$. Two other modes have the ferromagnetic character and were completely out of the focus of interest of researchers.

However, the normal modes of the uniform precession are only partial solutions of equations of motion of uniform magnetizations of sublattices, while the general solution of these equations should contain nutations at the background of the uniform precession in analogy with problems of a sphere pendulum and a heavy gyroscope [21]. The continuous energy spectrum of nutations of magnetizations of sublattices accompanying the normal modes of their regular precession for ideal antiferromagnetic nanoparticles has been recently described in [22]. In fact, the presence of the excitation branch corresponding to one of the ferromagnetic normal modes with the local energy minimum for the vectors of sublattice magnetizations precessing in the equatorial plane and nutations of magnetizations accompanying this normal mode gives the phenomenological explanation of macroscopic quantum effects observed in Mössbauer absorption spectra [9-13] and described in the quantum-mechanical model of antiferromagnetic nanoparticles $[7,8,14]$.

The continuous models described in $[18,21]$ are the basis for further development of the theory of nanoparticles with different magnetic natures which can be implemented as the method for quantitative analysis of experimental data, in particular, a large array of the Mössbauer spectra of nanoparticles measured for the last half-century [23]. Taking into account the general validity of the Néel idea, the next stage on the way to solve this problem is a generalization of these continuous models developed for ideal antiferromagnetic nanoparticles for the case of the presence of an uncompensated magnetic moment, in particular, for studying the effect of the latter on the excitation spectrum and the shape of Mössbauer spectra of an ensemble of "uncompensated" antiferromagnetic nanoparticles and its temperature evolution. This study was aimed at solving these problems.

\section{Excitation Spectrum of Antiferromagnetic Nanoparticles}

In analogy with $[18,22]$, let us start the analysis with the simplest expression for the energy density of an antiferromagnetic particle with the exchange interaction constant $J>$ 0 , the constant of the axial magnetic anisotropy $K$ in the approximation of two sublattices with magnetizations $\mathbf{M}_{1}$ and $\mathbf{M}_{2}$ :

$$
E=J \mathbf{M}_{1} \mathbf{M}_{2}-\frac{K}{2}\left(\cos ^{2} \theta_{1}+\cos ^{2} \theta_{2}\right) .
$$

Here, $\theta_{1}$ and $\theta_{2}$ are angles between vectors $\mathbf{M}_{1}$ and $\mathbf{M}_{2}$ and the easy axis. The only difference from the case of ideal antiferromagnetic particles $[18,22]$ is that the absolute values of magnetizations $M_{1}$ and $M_{2}$ in (1) can be not equal. We have neglected distinction of magnetic anisotropy for two sublattices in (1) because of small values of uncompensated magnetic moment.

In accordance with the classical theory of the antiferromagnetic resonance $[19,20]$ and ferromagnetic resonance [24], the phenomenological consideration can be performed within the assumption that the magnetic moment of each $i$ th sublattice precesses in the internal effective field:

$$
\mathbf{H}_{i}^{(\mathrm{eff})}=-\frac{\partial E}{\partial \mathbf{M}_{i}}
$$

and equations of motion for the sublattice magnetizations can be presented in the following form:

$$
\dot{\mathbf{M}}_{i}=-\gamma_{i}\left[\mathbf{M}_{i}, \mathbf{H}_{i}^{(\mathrm{eff})}\right] .
$$

Here, $\gamma_{i}$ is the magnetomechanical ratio for $i$ th sublattice. In our case effective magnetic fields acting on each of sublattices are determined by expressions

$$
\begin{aligned}
& \mathbf{H}_{1}^{(\text {eff })}=-H_{E 2} \mathbf{m}_{2}+H_{A 1} m_{1 z} \mathbf{n}_{z}, \\
& \mathbf{H}_{2}^{(\text {eff })}=-H_{E 1} \mathbf{m}_{1}+H_{A 2} m_{2 z} \mathbf{n}_{z} .
\end{aligned}
$$

Here, we have introduced effective values of the exchange field, $H_{E i}=J M_{i}$, and the anisotropy field, $H_{A i}=K / M_{i}$, in accordance with $[19,20,24]$ as well as normalized values of magnetizations of sublattices $\mathbf{m}_{i}=\mathbf{M}_{i} / M_{i}$ and their projections $m_{i z}=M_{i z} / M_{i}$ on the easy axis with the unit vector $\mathbf{n}_{z}$ for $i$ th sublattice.

It is convenient to look for axially symmetric solutions of equations of motion (3) in the form of the self-consistent and uniform precession of vectors $\mathbf{M}_{1}$ and $\mathbf{M}_{2}$ around the easy axis:

$$
\begin{aligned}
& m_{i x}(t)=m_{i \perp} \cos \omega t, \\
& m_{i y}(t)=m_{i \perp} \sin \omega t .
\end{aligned}
$$

Substituting these expressions into equations of motion (3), one comes to the combined equations for the components of the sublattice magnetizations

$$
\begin{aligned}
m_{1 \perp}\left(\beta \widetilde{\omega}-m_{2 z}+k m_{1 z}\right) & =-m_{1 z} m_{2 \perp} \\
m_{2 \perp}\left(\widetilde{\omega}-m_{1 z}+k m_{2 z}\right) & =-m_{2 z} m_{1 \perp} .
\end{aligned}
$$




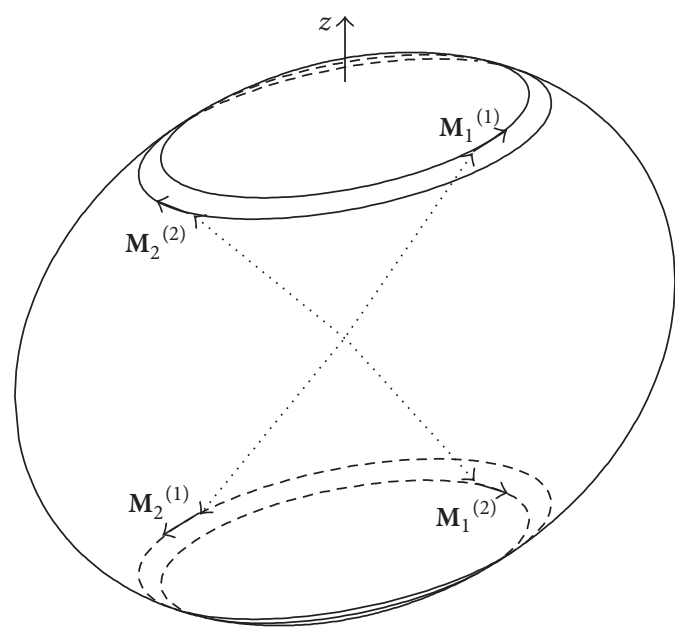

(a)

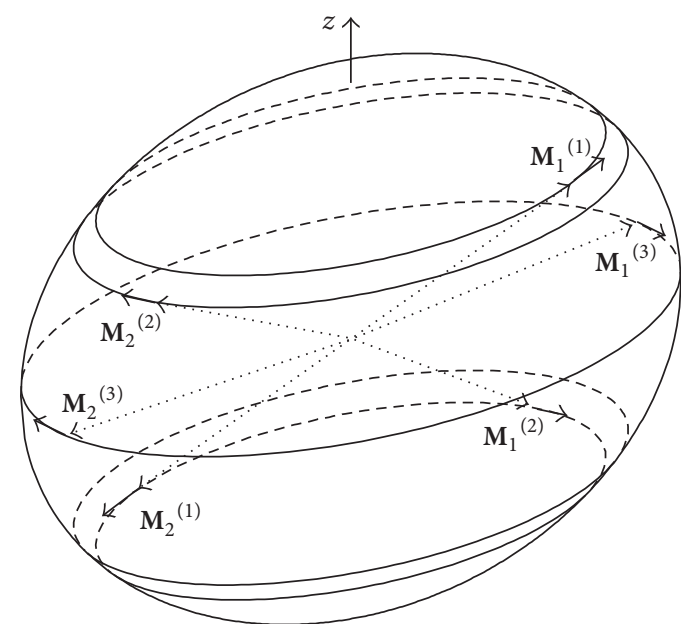

(b)

FIgURE 1: Normal modes of the self-consistent precession of the sublattice magnetization vectors $\mathbf{M}_{1}$ and $\mathbf{M}_{2}$ of an ideal antiferromagnetic particle for $E^{\prime}=E / A=\left(\right.$ a) -1.003 and (b) -0.997 with $m= \pm m_{\max }(E)$. Hereinafter, all calculations were performed for $k=K / A=0.01$.

Here, we have introduced the ratio of the sublattice spins

$$
\beta=\frac{S_{1}}{S_{2}} \equiv \frac{\gamma_{2} M_{1}}{\gamma_{1} M_{2}},
$$

normalized values of the magnetization precession frequency

$$
\widetilde{\omega}=-\frac{\omega}{J \sqrt{\beta \gamma_{1} \gamma_{2} M_{1} M_{2}}}
$$

and the anisotropy constant

$$
k=\frac{K}{A}
$$

defined by the exchange interaction energy density

$$
A=J M_{1} M_{2}
$$

Further without loss of a community, we will consider that $\beta \leq 1$ and the normalized value of uncompensated magnetic moment $1-\beta \ll 1$ in the case of the Néel ensemble of antiferromagnetic nanoparticles.

The solutions of (6a) and (6b) are four normal modes of self-consistent and homogeneous precession of the vectors $\mathbf{M}_{1}$ and $\mathbf{M}_{2}$ around the easy axis (see Figure 1). These modes are defined by parametric relations between the components of the sublattice magnetizations, $m_{1 z}$ and $m_{2 z}$, which are rather complicated for perception so that we represent only the equation for the normalized precession frequency

$$
\begin{aligned}
2 \beta \widetilde{\omega} & =(\beta-k) m_{1 z}+(1-\beta k) m_{2 z} \\
& \pm \sqrt{\left[(\beta+k) m_{1 z}+(1+\beta k) m_{2 z}\right]^{2}-4 k\left(1+\beta^{2}+\beta k\right) m_{1 z} m_{2 z}} .
\end{aligned}
$$

Analysis of equations like (11) in physically meaningful limiting cases of small anisotropy energy as compared to the exchange energy $(k \ll 1)$ and small deviations of the vectors $\mathbf{M}_{1}$ and $\mathbf{M}_{2}$ from the easy axis allows one to describe the antiferromagnetic resonance $[19,20]$ and ferromagnetic resonance [24] on a phenomenological level. However, solution of (6a) and (6b) in a general form allows one to find new qualitative features of the thermodynamics of an ensemble of antiferromagnetic nanoparticles $[18,22]$ and to develop a technique for quantitative treatment of the experimental data [17].

2.1. Normal Precession Modes for Ideal Antiferromagnetic Nanoparticles. For ideal antiferromagnetic particles $(\beta=1$, $M_{1}=M_{2}$ ) the combined equations (6a) and (6b) are reduced to the following equation for the components of the sublattice magnetizations:

$$
\left[m_{1 \perp} m_{2 \perp}(1+k)-1-m_{1 z} m_{2 z}\right]\left(m_{1 z}-m_{2 z}\right)=0 .
$$

Here is a simple analytical solution as a set of four normal precession modes (Figure 1) at the frequencies

$$
\widetilde{\omega}=\frac{(1-k) m}{2} \pm \frac{1}{2} \sqrt{(1+k)^{2} m^{2}-4 k(2+k) m_{1 z} m_{2 z}},
$$

where

$$
m=m_{1 z}+m_{2 z}
$$

is the normalized projection of total magnetization onto the anisotropy axis. As follows from (12), these modes are characterized by the following relations between the components of the sublattice magnetizations [18]:

$$
\begin{aligned}
& m_{2 z}^{(1,2)}=\frac{-m_{1 z} \pm\left(1-m_{1 z}^{2}\right)(1+k) \sqrt{k(2+k)}}{1+\left(1-m_{1 z}^{2}\right) k(2+k)} \\
& m_{2 z}^{(3,4)}=m_{1 z} \equiv m_{z} .
\end{aligned}
$$

Here, for the first three modes, the transverse components of the vectors $\mathbf{M}_{1}$ and $\mathbf{M}_{2}$ have opposite signs (see Figure 1) and 
the vectors $\mathbf{M}_{1}$ and $\mathbf{M}_{2}$ for the fourth mode coincide with each other. Relations (15a) and (15b) are illustrated in Figure 2 for $\beta=1$.

The normal modes correspond to four excitation branches in the energy spectrum of ideal antiferromagnetic particles (Figure 2, $\beta=1$ ) [18]:

$$
\begin{aligned}
\frac{E_{1,2}}{A} & =-\frac{1-k m_{1 z} m_{2 z}}{1+k}-\frac{k}{2}\left(m_{1 z}^{2}+m_{2 z}^{2}\right), \\
\frac{E_{3}}{A} & =-1+(2-k) m_{z}^{2}, \\
\frac{E_{4}}{A} & =1-k m_{z}^{2} .
\end{aligned}
$$

These excitation branches are shown in two right upper panels of Figure 2. All the normal modes (15a) and (15b) and the excitation energy branches (16a)-(16c) are symmetric with respect to the permutation of the vectors $\mathbf{M}_{1}$ and $\mathbf{M}_{2}$. An informal feature of the energy spectrum of the ensemble of ideal antiferromagnetic particles is the presence of six bifurcation points where the directions of the vectors $\mathbf{M}_{1}$ and $\mathbf{M}_{2}$ for different excitation branches coincide (Figure 2, $\beta=1$ ) and their projections are

$$
\begin{aligned}
& m_{z b}^{(1,2)}= \pm 1 \\
& m_{z b}^{(1,3)}=-m_{z b}^{(2,3)}=\sqrt{\frac{k}{(2+k)}} \\
& m_{z b}^{(3,4)}= \pm 1
\end{aligned}
$$

Note that two low-lying and weakly split excitation branches (corresponding to the normal modes 1 and 2) meet completely the doublet pattern of the low-lying levels in the energy spectrum obtained in the quantum-mechanical calculations $[7,8]$ for ideal antiferromagnetic particles with rather large values of the spin $S$ and the constant $k$ (or to be more precise for $k S^{2} \gg 1$ ). Substituting relations (15a) and (15b) into the initial equations (6a) and (6b), one defines also the normalized precession frequencies for each of the normal modes

$$
\begin{aligned}
\widetilde{\omega}_{1,2} & =m_{2 z}-m_{1 z}\left(k-\frac{1+m_{1 z} m_{2 z}}{(1+k)\left(1-m_{1 z}^{2}\right)}\right), \\
\widetilde{\omega}_{3} & =(2-k) m_{z}, \\
\widetilde{\omega}_{4} & =-k m_{z} .
\end{aligned}
$$

Remaining within the generally accepted assumption on the smallness of the anisotropy energy with respect to the exchange energy

$$
k \ll 1 \text {, }
$$

for two low-lying excitation branches corresponding to the normal modes 1 and 2, one finds approximate expressions for the precession frequencies (18a) of the vectors $\mathbf{M}_{1}$ and $\mathbf{M}_{2}$ in these modes:

$$
\widetilde{\omega}_{1,2} \approx \pm \sqrt{k(2+k)}\left\lfloor 1-k\left(1-m_{1 z}^{2}\right)\right\rfloor .
$$

The modes given by (15a) correspond to the classical theory of antiferromagnetic resonance in zero magnetic field at the frequency $[19,20]$

$$
\omega_{1,2}= \pm \omega_{0}=\mp \gamma H_{0} \equiv \mp \gamma \sqrt{H_{A}\left(2 H_{E}+H_{A}\right)} .
$$

Note that (20b) has been obtained within the approximation of small deviations of the vectors $\mathbf{M}_{1}$ and $\mathbf{M}_{2}$ from the easy axis $[19,20]$, whereas (20a) confirms that the classical equation (20b) is valid for all possible orientations of the vectors $\mathbf{M}_{1}$ and $\mathbf{M}_{2}$ to within small terms of an order of $k$. That means that when condition (19) holds, within the same accuracy the antiferromagnetic resonance in zero magnetic field is similar to the conventional resonance methods in a constant magnetic field with the strength $H_{0}$. With that the normal mode 3 is characterized by the "exchange" precession frequency $\omega_{E}=\gamma H_{E}$ :

$$
\omega_{3}=-\gamma H_{E}(2-k) m_{z}
$$

and mode 4 is characterized by the lower "ferromagnetic" frequency $\omega_{A}=\gamma H_{A}$ :

$$
\omega_{4}=\gamma H_{A} m_{z}
$$

that is, the characteristic frequencies depend essentially on the orientation of the vectors $\mathbf{M}_{1}$ and $\mathbf{M}_{2}$ as in the case of ferromagnetic particles [23].

Since the middle of the 20th century, modes 3 and 4 have fallen beyond the focus of interest of researchers primarily because of the approximation of small deviations of the vectors $\mathbf{M}_{1}$ and $\mathbf{M}_{2}$ from the easy axis used in the theory of antiferromagnetic resonance and because these "ferromagnetic" modes lie much higher in energy than antiferromagnetic modes 1 and 2 (Figure 2). However, even a brief glance at the right lower (for $\beta=1$ ) panel of Figure 2 reveals that the excitation branch corresponding to mode 3 must be taken into account in the description of the temperature dependence of spectroscopic data, for instance, Mössbauer spectra. Moreover, one can understand even without any numerical calculations that the inclusion of this excitation branch with the local energy minimum for the vectors $\mathbf{M}_{1}$ and $\mathbf{M}_{2}$ precessing in the equatorial plane should lead to the emergence of a single line (or a quadrupolar doublet) with a low hyperfine field $H_{\mathrm{hf}}$ value in the Mössbauer spectrum against the background of the hyperfine structure (corresponding to modes 1 and 2) with high $H_{\mathrm{hf}}$ value. This statement is in fact a phenomenological explanation of the macroscopic quantum effects observed earlier in the absorption spectra [9-13]. According to (15a)-(16c), mode 4 is of critical value in the case of metamagnetism at $k>2$ (see [25]) and its consideration is beyond the scope of the present publication.

2.2. Normal Precession Modes for "Uncompensated" Antiferromagnetic Nanoparticles. In the presence of uncompensated magnetic moment in antiferromagnetic particles $(\beta<1)$ the combined equations (6a) and (6b) can be reduced to 

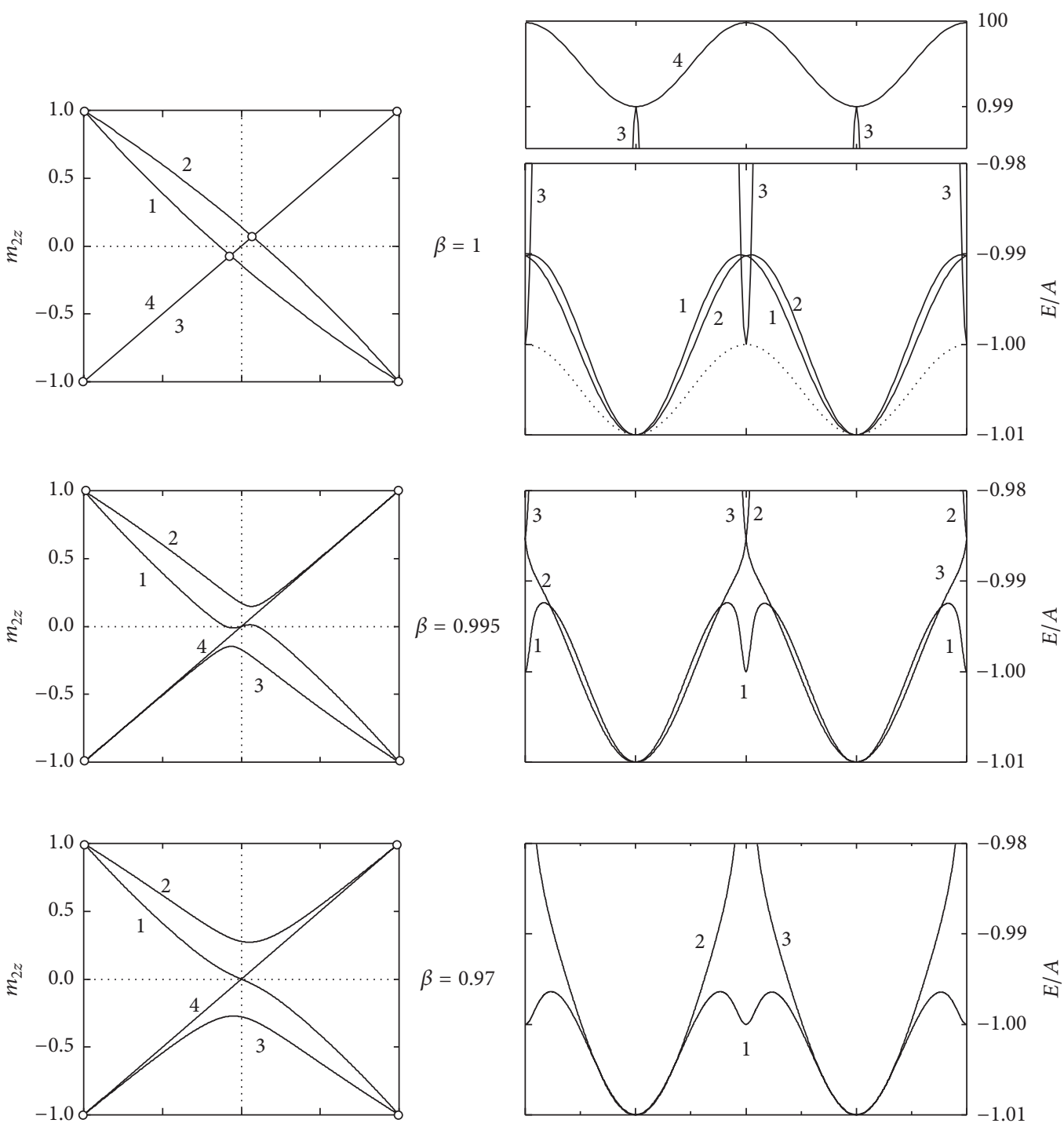

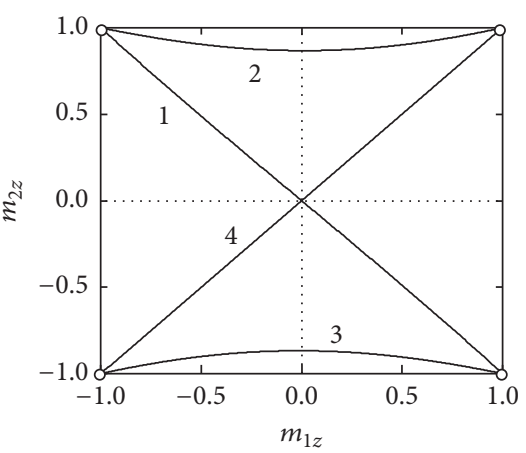

(a)
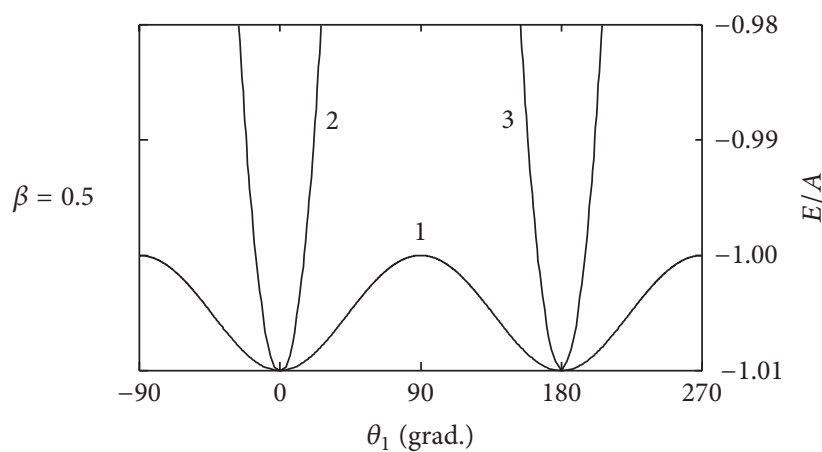

(b)

FIgURE 2: (a) Correlation between projections of the sublattice magnetization vectors $m_{1 z}$ and $m_{2 z}$ onto the easy axis for four normal precession modes of the vectors $\mathbf{M}_{1}$ and $\mathbf{M}_{2}$ of an ideal antiferromagnetic particle $(\beta=1)$ and "uncompensated" antiferromagnetic particles $(\beta<1)$ at $k=0.01$. Branching points are indicated by the circles. (b) Four excitation branches for $\beta=1$ and three low-lying excitation branches $\beta<1$ (solid lines) in the energy spectrum of antiferromagnetic particles depending on the angle of deviation of the vector $\mathbf{M}_{1}$ from the easy axis. The dotted line shows the energy relief for the corresponding ferromagnetic particle. 
the following equation for the components of the sublattice magnetizations:

$$
\begin{aligned}
& m_{1 \perp} m_{2 \perp}\left[(\beta+k) m_{1 z}-(1+\beta k) m_{2 z}\right] \\
& =-m_{1 z} m_{2 \perp}^{2}+\beta m_{2 z} m_{1 \perp}^{2} .
\end{aligned}
$$

There is no such simple analytical solution like that for (12) for ideal antiferromagnetic particles. However, this equation can be reduced to a fourth-order equation and the solution of the latter allows one to find the parametric relations between the longitudinal components of magnetization sublattice vectors $m_{1 z}$ and $m_{2 z}$ as well as the corresponding excitation branches in the energy spectrum. The results from such calculations are shown in the lower panels $(\beta<1)$ of Figure 2 . As can be seen in Figure 1, the general character of the solution in the form of four normal modes of the self-consistent and uniform precession of the magnetization vectors $\mathbf{M}_{1}$ and $\mathbf{M}_{2}$ around the easy axis is also retained when there is an uncompensated moment in antiferromagnetic particles. We perform also such calculations for rather large values of uncompensated magnetic moment $(\beta=0.5)$ which formally corresponds to ferrimagnetic particles. The latter case will be considered briefly in Section 4.

At the same time, the excitation branches in the energy spectrum that correspond to normal precession modes change essentially their form already at very low values of the uncompensated moment (Figure 2, $\beta=0.995$ ). The bifurcation points (17b) disappear and a peculiar hybridization of the excitation branches characteristic of ideal antiferromagnetic particles then takes place. With that branches 1 and 4 remain symmetric relative to permutation of $\mathbf{M}_{1}$ and $\mathbf{M}_{2}$ while the shape of branch 1 acquires that of a three-well potential with absolute energy minima for the mutually opposite orientation of vectors $\mathbf{M}_{1}$ and $\mathbf{M}_{2}$ along each of two directions along the easy axis and with the local energy minimum that corresponds to the precession of these vectors in the equatorial plane. Branches 2 and 3 for $\beta<1$ remain symmetrical relative to the simultaneous substitution of projections $m_{1 z}$ and $m_{2 z}$ for $-m_{1 z}$ and $-m_{2 z}$, and the orientation of vector $\mathbf{M}_{1}$ with a smaller value of magnetic moment varies for these branches over the entire range of possible values of polar angle $\theta(-1 \leq$ $\left.m_{1 z} \leq 1\right)$, while the vector $\mathbf{M}_{2}$ is reoriented in the limited range of $\theta\left(m_{2 z}^{(2)} \geq m_{z c}\right.$ and $m_{2 z}^{(3)} \leq-m_{z c}$, where $\left.m_{z c}>0\right)$ as seen in Figure 2. Each of the excitation branches has one energy minimum for the mutually opposite orientation of vectors $\mathbf{M}_{1}$ and $\mathbf{M}_{2}$ along each of two directions along the easy axis and one maximum for the opposite orientation of both the vectors $\mathbf{M}_{1}$ and $\mathbf{M}_{2}$ (Figure 2).

Note that for $\beta<1$ three low-lying excitation branches corresponding to the three normal modes and weakly split within the macroscopic energy barrier $(E<-A)$ meet completely the quadruplet pattern (or weakly split pair of the Kramer's doublets) of the low-lying levels in the energy spectrum from the quantum-mechanical calculations for antiferromagnetic particles with uncompensated spin and rather large values of the scaling parameter $k S^{2} \gg 1$ (see Fig. 1 in [14]).
2.3. Nutations of Sublattice Magnetizations for Ideal Antiferromagnetic Nanoparticles. Modes of the uniform precession of magnetizations of sublattices described in the previous sections are partial solutions of the combined equations of motion (3). The general solutions are described by a system of differential equations for the longitudinal and transverse components of magnetizations of antiferromagnetic particles. In particular, the longitudinal components for ideal antiferromagnetic particles are given by the expression [22]

$$
d m_{1 z}=-d m_{2 z}= \pm \omega_{E} \sqrt{F\left(m_{1 z}, m_{2 z}, E\right)} d t
$$

Here,

$$
\begin{aligned}
F\left(m_{1 z}, m_{2 z}, E\right) & \\
= & 1-E^{\prime 2}-\left(1+k E^{\prime}\right)\left(m_{1 z}^{2}+m_{2 z}^{2}\right) \\
& +2 m_{1 z} m_{2 z}\left(E^{\prime}+\frac{k}{2}\left(m_{1 z}^{2}+m_{2 z}^{2}\right)\right) \\
& -\frac{k^{2}}{4}\left(m_{1 z}^{2}+m_{2 z}^{2}\right)^{2}
\end{aligned}
$$

and $E^{\prime}=E / A$. In complete agreement with the axial symmetry of the problem, (23a) indicates that projection (14) of the total magnetic moment on the anisotropy axis, as well as the energy, is an integral of motion. Then, it is convenient to rewrite (23a) and (23b) for the longitudinal component of the antiferromagnetic vector

$$
l_{z}=m_{1 z}-m_{2 z}
$$

The change of variables results in the following equation:

$$
d l_{z}= \pm \frac{\omega_{0}}{2} \sqrt{\left(l_{z}^{2}-l_{1}(E, m)\right)\left(l_{2}(E, m)-l_{z}^{2}\right)} d t
$$

Here, omitting the notation of the functional dependence on $E$ and $m$,

$$
\begin{aligned}
l_{1,2} & =l_{0} \pm \Delta l=-4 \frac{1+E^{\prime}(1+k)+k^{2} m^{2} / 4 \pm \sqrt{s}}{k(2+k)}, \\
s & =\left(1+k+E^{\prime}\right)^{2}+\frac{k^{2} m^{4}}{4}-k m^{2}\left(1-E^{\prime}\right) .
\end{aligned}
$$

Equation (25) determines the time dependence and the range of variation of the longitudinal components of sublattice magnetizations. These components in turn determine the type and characteristics of trajectories of motion of the vectors $\mathbf{M}_{1}$ and $\mathbf{M}_{2}$ in the form of nutations [21], that is, the self-consistent precession of these vectors about the easy axis with the simultaneous oscillations in the polar angle in the range given by the parameters $l_{1}$ and $l_{2}$. The resulting trajectories of motion for given $E$ and $m$ values are also determined by the parametric relations between the transverse and longitudinal components of sublattice magnetizations. For 
example, the time variation of the azimuth angle of the vector $\mathbf{M}_{1}$ is described by the following equation:

$$
\begin{aligned}
d \varphi_{1} & =\omega_{A}\left[\frac{m_{1 z}}{1-m_{1 z}^{2}}\left(E^{\prime}-\frac{(1-k) m^{2}-(1+k) l_{z}^{2}}{4}\right)\right. \\
& \left.-m+(1+k) m_{1 z}\right] d t .
\end{aligned}
$$

Then, self-consistent trajectories of motion of the vectors $\mathbf{M}_{1}$ and $\mathbf{M}_{2}$ are given by (1), (14), and (24)-(27). Examples of calculations of such trajectories are given in Figure 3, where several initial nutations of the vectors $\mathbf{M}_{1}$ and $\mathbf{M}_{2}$ over the surface of the sphere with the radius $\mathbf{M}_{0}$ are shown. In the general case, these trajectories are not closed; that is, each of the vectors infinitely often passes via the minimum and maximum values of the projections $m_{1 z}$ and $m_{2 z}$ [21]. Since the period of the high-frequency nutations is much less than the characteristic times in the majority of experimental techniques for studying antiferromagnetic particles (in particular, in Mössbauer spectroscopy, not speaking about magnetization curves), we are interested in the average (over the nutation period) values of longitudinal components of magnetizations of sublattices given by (25). This equation will be analyzed in detail further.

If condition (19) holds, the range of allowed $m$ values for a given energy is determined by the condition of positivity of the parameter $s$ :

$$
m^{2} \leq m_{\max }^{2} \equiv 2 \frac{1-E^{\prime}-\sqrt{-(2+k)\left(k+2 E^{\prime}\right)}}{k}
$$

(see Figure 4). In turn, according to (25), the range of allowed $l_{z}$ values for given $E$ and $m$ values is determined by the condition

$$
l_{1} \leq l_{z}^{2} \leq l_{2}
$$

As seen from (26a) and (26b) and Figures 3 and 4, the character of nutations is different in three energy ranges. When

$$
E^{\prime}=\frac{E}{A} \leq-1,
$$

the minimum and maximum (in absolute value) $l_{z}$ values for a given energy, as well as the maximum range of nutations over the polar angle, are achieved at $m=0$ (Figures 3(a) and 4):

$$
\begin{aligned}
& l_{\text {min }}(E)=\sqrt{l_{1}(E, 0)}, \\
& l_{\text {max }}(E)=\sqrt{l_{2}(E, 0)} .
\end{aligned}
$$

The range of nutations for a given energy decreases with an increase in the absolute value of $m$ up to the maximum (in absolute value) projection of the total moment, $m=$ $\pm m_{\max }(E)$, when $s=0, l_{1}=l_{2}=l_{0}$, and the normal precession modes of sublattice magnetizations 1 and 2 are implemented. According to (14), (24), and (25), the average (over the period of nutations $T_{E, m}$ ) values of longitudinal components of sublattice magnetizations in this energy range for the given $E$ and $m$ values are determined by the average value $\left\langle l_{z}\right\rangle_{T_{E, m}}$ :

$$
\bar{m}_{1 z, 2 z}(E, m)=\frac{m}{2} \pm\left\langle l_{z}\right\rangle_{T_{E, m}}=\frac{m}{2} \pm \frac{\pi}{\omega_{0} T_{E, m}} .
$$

Here, the period of nutations for the given $E$ and $m$ is

$$
T_{E, m}=\frac{4}{\omega_{0} \sqrt{l_{2}}} I_{1}(\gamma)
$$

and defined by the complete elliptic integral of the first kind

$$
I_{1}(\gamma)=\int_{\gamma}^{1} \frac{d x}{\sqrt{\left(x^{2}-\gamma^{2}\right)\left(1-x^{2}\right)}}
$$

$\gamma=\sqrt{l_{1} / l_{2}}$

Let us consider now the next energy range, where three normal precession modes of the sublattice magnetizations coexist (see the uppermost panels of Figures 2 and 4):

$$
-1 \leq E^{\prime} \leq E_{b}^{\prime(1,3)} .
$$

Here,

$$
E_{b}^{\prime(1,3)}=E_{b}^{\prime(2,3)} \equiv-1+k \frac{2-k}{2+k}
$$

correspond to the branching points $(17 \mathrm{a})-(17 \mathrm{c})$ at which the directions of the vectors $\mathbf{M}_{1}$ and $\mathbf{M}_{2}$ for excitation branches 1 and 3 (or 2 and 3 ) are the same. Two situations are possible in this energy range (Figures $3(\mathrm{~b})-3(\mathrm{~d})$ and 4 ). For a given $E^{\prime}$ value from the range (33a), there is always the value

$$
m= \pm m_{3}(E) \equiv \pm 2 \sqrt{\frac{1+E^{\prime}}{2-k}}
$$

which corresponds to the normal precession mode 3 , and in this case $l_{1}\left(E, m_{3}(E)\right)=0$. Then, if

$$
m_{3}(E) \leq|m| \leq m_{\max }(E),
$$

the previous situation (for $E^{\prime} \leq-1$ ) is implemented, in which (31a)-(31b) and (32a)-(32c) are valid (see Figure 3(c)). Another situation takes place at

$$
0 \leq|m| \leq m_{3}(E)
$$

when $l_{1}(E, m)<0$ and in accordance with (25) nutations become symmetric with respect to the change of the sign of $l_{z}$ in the interval

$$
-\sqrt{l_{2}(E, m)} \leq l_{z} \leq \sqrt{l_{2}(E, m)}
$$

(see Figures 3(b) and 3(d)). Then $\left\langle l_{z}\right\rangle_{T_{E, m}}=0$ and

$$
\bar{m}_{1 z}(E, m)=\bar{m}_{2 z}(E, m)=\frac{m}{2} .
$$




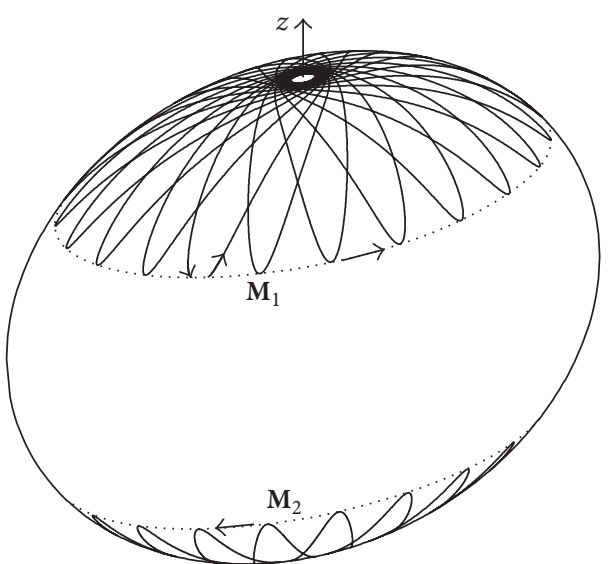

(a)

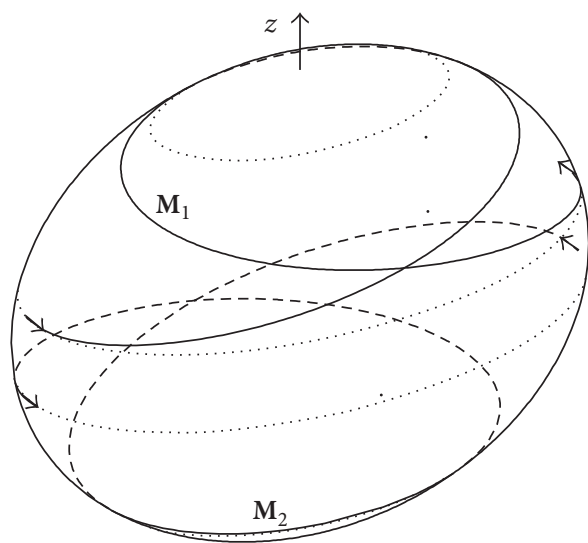

(c)

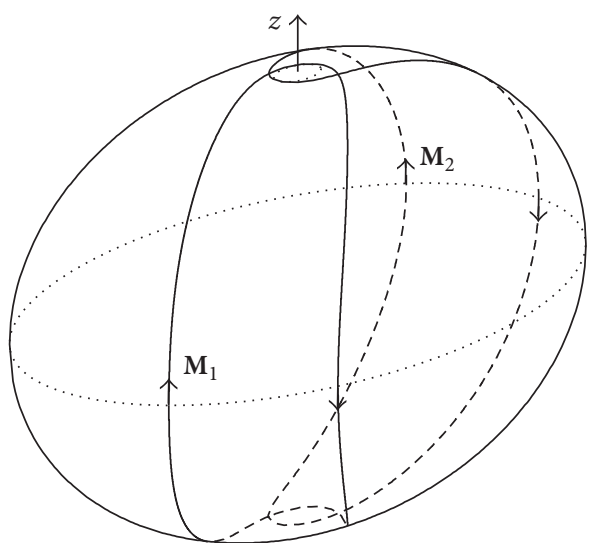

(b)

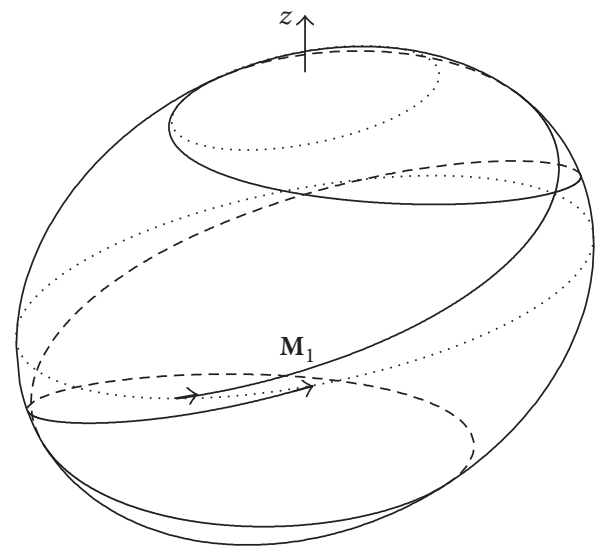

(d)

FIGURE 3: Dissipationless trajectories of motion of sublattice magnetizations $\mathbf{M}_{1}$ and $\mathbf{M}_{2}$ for an ideal antiferromagnetic particle. Nutations of the magnetization vectors with $m=0$ for $E^{\prime}=E / A=(\mathrm{a})-1.003$ and (b) -0.999 . Nutations of the magnetization vectors with values $m$ $=$ (c) 0.08 and (d) 0.075 for $E^{\prime}=-0.999$. Panel (a) shows 23 initial nutations whereas panels (b)-(d) show the first nutations. Points show minimum and maximum values of projections $m_{1 z}$ and $m_{2 z}$ during nutations.

The regions in which these two situations are implemented are separated in Figure 4 by curve $l_{3}$.

Finally, in the third energy range

$$
E_{b}^{(1,3)} \leq E^{\prime} \leq-\frac{k}{2},
$$

the range of possible $m$ values unlike (28) is limited by the projection of the total moment for the normal precession mode 3:

$$
|m| \leq m_{3}(E) .
$$

In these $E$ and $m$ ranges, nutations of the vectors $\mathbf{M}_{1}$ and $\mathbf{M}_{2}$ are always symmetric with respect to the sign of $l_{z}$ in range (36), and the average projections of magnetizations of sublattices are determined by (37). Note that the upper limit in (38) is necessary for the formal fulfillment of condition (28), though energies of such order of magnitude under the fulfillment of condition (19) are attainable only at unrealistically high temperatures.
2.4. Nutations of Sublattice Magnetizations for "Uncompensated" Antiferromagnetic Nanoparticles. In the presence of uncompensated magnetic moment in antiferromagnetic particles $(\beta<1)$ the differential equation (23a) for the longitudinal components of ideal antiferromagnetic particles change slightly in a form:

$$
\frac{d m_{1 z}}{\omega_{E 1}}=-\frac{d m_{2 z}}{\omega_{E 2}}= \pm \sqrt{F\left(m_{1 z}, m_{2 z}, E\right)} d t .
$$

Here, $\omega_{E 1,2}=-\gamma H_{E 2,1}$. Again the total magnetic moment on the anisotropy axis, as well as the energy, is an integral of motion, which can be written in the following form:

$$
m=\beta m_{1 z}+m_{2 z}
$$

However, there is no such simple analytical solution for normal precession modes and nutations of the sublattice magnetizations like that in the previous section. Instead one can define characteristics of the normal precession modes 

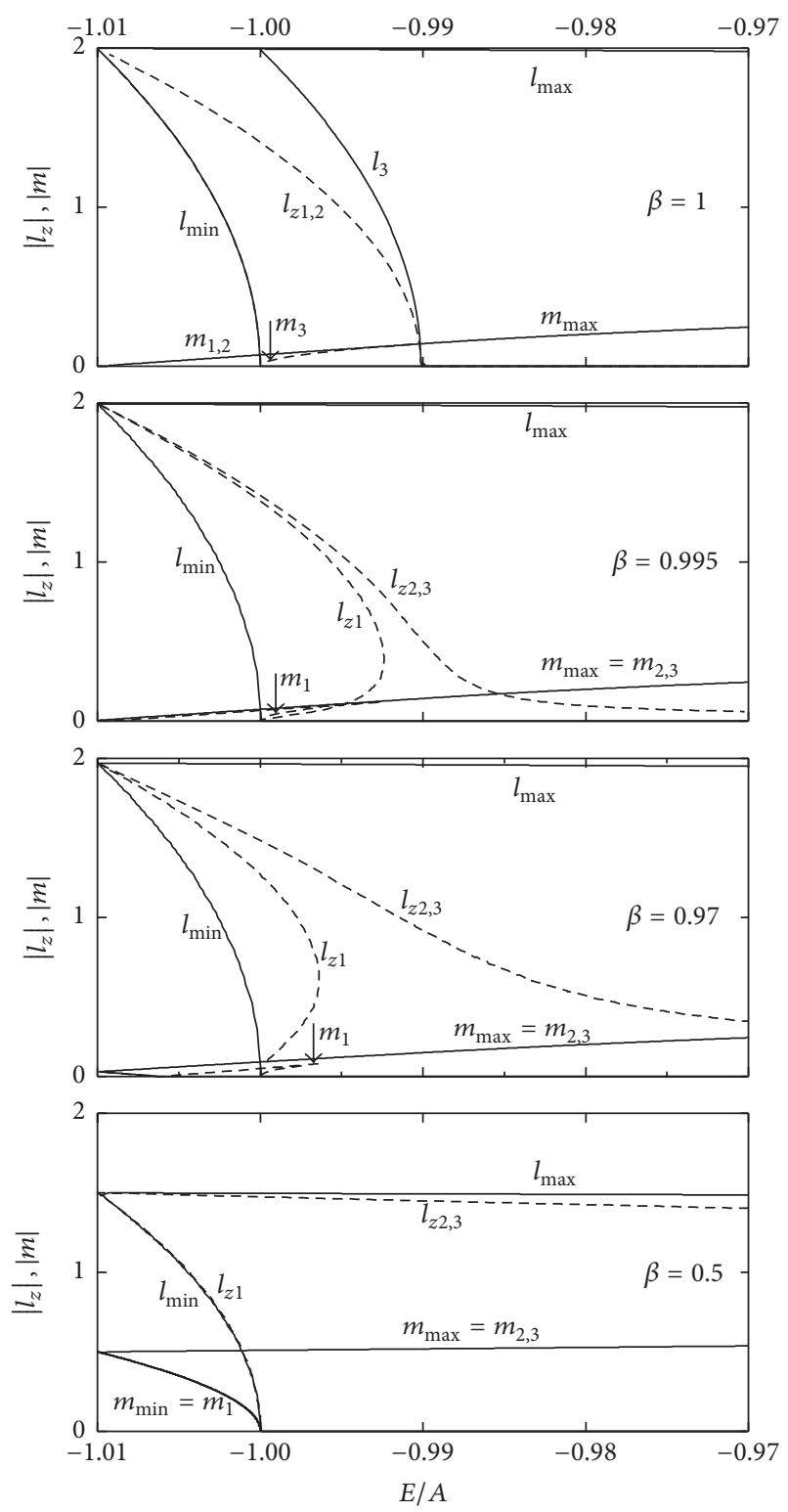

FIgURE 4: Ranges of the allowed projections of the total magnetic moment $m$ and the vector of antiferromagnetism $l_{z}$ on the anisotropy axis for ideal $(\beta=1)$ and "uncompensated" $(\beta<1)$ antiferromagnetic particles (see main text). Dashed lines $l_{z 1}, l_{z 2}$, and $l_{z 3}$ correspond to the normal uniform precession modes of magnetizations of sublattices $\mathbf{M}_{1}$ and $\mathbf{M}_{2}$.

and nutations for given $E$ and $m$ values by means of solution of the fourth-order equation

$$
F\left(m_{1 z}, m-\beta m_{1 z}, E\right)=0 .
$$

Nevertheless, forms and characteristics of self-consistent trajectories of motion of the vectors $\mathbf{M}_{1}$ and $\mathbf{M}_{2}$ in both the cases are similar in many respects. Ranges of the allowed projections of the total magnetic moment $m$ and the vector of antiferromagnetism

$$
l_{z}=\beta m_{1 z}-m_{2 z}
$$

on the anisotropy axis for "uncompensated" $(\beta<1)$ antiferromagnetic particles have been calculated by solving (42) and shown in lower panels of Figure 4 . As seen in the figure, the general character of the solution in the form of three (at lower energy values) normal modes of self-consistent and regular precession of the vectors $\mathbf{M}_{1}$ and $\mathbf{M}_{2}$ around the easy axis, which are accompanied by nutations, is again retained when there is an uncompensated moment in antiferromagnetic particles.

First of all, at $\beta<1$, ranges of the allowed projections $m$ (in absolute value) are restricted to the lower limit

$$
|m| \geq m_{\min }(E)
$$

in the energy minima, the only allowed values $m(-A-$ $K)= \pm(1-\beta)$, and at energy values higher than some value $E_{0}(\beta, k), m_{\min }(E)=0$ (see Figure 4 ). The normal precession modes of sublattice magnetizations 2 and 3 are implemented at the maximum (in absolute value) projections of the total magnetic moment, $m= \pm m_{\max }(E)$. The character of nutations of the vectors $\mathbf{M}_{1}$ and $\mathbf{M}_{2}$ is different in three energy ranges like that for ideal antiferromagnetic particles.

At $E^{\prime} \leq-1$ there are two possible types of the solution of (42). The minimum and maximum (in absolute value) $l_{z}$ values for a given energy, as well as the maximum range of nutations over the polar angle, are achieved at $m= \pm m_{\min }(E)$. Unlike ideal antiferromagnetic particles, the limiting $l_{z}$ values for a given energy in the range (30) and the relevant range of nutations appear to be different in two potential energy wells, which correspond to two different pairs of real roots of (42). However, the symmetry relative to the simultaneous replacement of signs of $m$ and $l_{z}$ (or $m_{1 z}$ ) remains. The range of nutations for a given energy decreases in both the potential energy wells with an increase in the absolute value of $m$ up to the value $m= \pm m_{1}^{(1)}(E)$, when the normal precession mode 1 is realized. With a further increase in the absolute value of $m$ nutations in the potential energy well corresponding to the normal precession mode 1 disappear (two complex roots of (42)) while the range of nutations in the other potential energy well (two real roots of (42)) decreases up to the value $m(E)= \pm m_{\max }(E)$ when the normal precession modes 2 and 3 are implemented (Figure 4 ).

Let us consider the next energy range, where also three normal precession modes of the sublattice magnetizations coexist (see Figures 2 and 4):

$$
-1 \leq E^{\prime} \leq E_{\max }^{\prime(1)}
$$

where $E_{\max }^{\prime(1)}$ corresponds to the energy maximum for the excitation branch 1 . Here are already three characteristic ranges of $m$ values. The maximum range of nutations extended in both the potential energy wells (two real and two complex roots of (42)) is realized at $m=0$. This type of solution for a given energy value in the range (45) retains with an increase in the absolute value of $m$ up to the value $m=$ $\pm m_{1}^{(2)}(E)$, when the normal precession mode 1 is realized in the vicinity of the local energy minimum of the excitation branch 1 (Figure 2). With a further increase in the absolute value of $m$ the behavior characteristic for the previous energy 
range $\left(E^{\prime} \leq-1\right)$ is observed: two ranges of nutations in both the potential energy wells and two different pairs of real roots of (42) up to the value $m= \pm m_{1}^{(1)}(E)$ followed by the only range of nutations (two real and two complex roots of (42)) with a further increase in the absolute value of $m$ up to the value $m= \pm m_{\max }\left(E^{\prime}\right)$.

Finally, in the third energy range

$$
E^{\prime} \geq E_{\max }^{\prime(1)}
$$

there are two real and two complex roots of (42) while nutations of the vectors $\mathbf{M}_{1}$ and $\mathbf{M}_{2}$ extend in both the potential energy wells for all possible $m$ values up to $|m|=$ $m_{\max }\left(E^{\prime}\right)$ when the normal precession modes 2 and 3 are implemented (Figure 4).

A detailed analysis of the functional dependence of the character of nutations on $E$ and $m$ in "uncompensated" antiferromagnetic particles is beyond the scope of this work and will be done elsewhere. As far as calculations of the average (over the period of nutations) values of longitudinal components of sublattice magnetizations for the given $E$ and $m$ values are concerned, one can use the general procedure for transforming an integral like that in (40) to a canonical form of elliptic integral of the first kind by reducing the polynomial of the fourth degree $F\left(m_{1 z}, m-\beta m_{1 z}, E^{\prime}\right)$ on $m_{1 z}$ with known roots to a product of two binomials on $m_{1 z}^{2}$. However, even consecutive records of the transformation occupy more than a half of the page while the final elliptic integrals all the same will be incomplete, that is, depending on two parameters. As an alternative, demanding the smaller number of elementary operations, one can calculate the average values of longitudinal components of sublattice magnetizations over the period of nutations in $j$ th range for the given $E$ and $m$ values by using the following equation:

$$
\bar{m}_{1 z}^{(j)}\left(E^{\prime}, m\right)=\frac{m_{1 z}^{(1)}+m_{1 z}^{(2)}}{2}+\Delta_{12} \frac{I_{2}\left(x_{0}, \delta_{0}\right)}{I_{3}\left(x_{0}, \delta_{0}\right)} .
$$

Here, $m_{1 z}^{(i)}$ are the roots of $(42)(i=1,2,3,4), x_{0}=\left(m_{1 z}^{(3)}+\right.$ $\left.m_{1 z}^{(4)}-m_{1 z}^{(1)}-m_{1 z}^{(2)}\right) /\left(2 \Delta_{12}\right)$, the parameter $\delta_{0}=\Delta_{34}^{2} / \Delta_{12}^{2}$ can be both positive (four real roots) and negative (two real and two complex roots) values, $\Delta_{i j}=\left(m_{1 z}^{(i)}-m_{1 z}^{(j)}\right) / 2$, and the following two-parameter integrals are introduced:

$$
\begin{aligned}
& I_{2}\left(x_{0}, \delta_{0}\right)=\int_{-1}^{1} \frac{x d x}{\sqrt{\left(1-x^{2}\right)\left[\left(x-x_{0}\right)^{2}-\delta_{0}\right]}}, \\
& I_{3}\left(x_{0}, \delta_{0}\right)=\int_{-1}^{1} \frac{d x}{\sqrt{\left(1-x^{2}\right)\left[\left(x-x_{0}\right)^{2}-\delta_{0}\right]}} .
\end{aligned}
$$

\section{Mössbauer Spectra of an Ensemble of Slowly Relaxing Antiferromagnetic Nanoparticles}

Now let us consider the effect of the nontrivial excitation spectrum of the Néel ensemble of antiferromagnetic nanoparticles with uncompensated magnetic moment (Figures 2 and 4) on the physically observable quantities by studying the shape of Mössbauer spectra of such an ensemble and its temperature evolution. We will stay within the simplest but physically justified limiting case of the slow (in comparison with the precession frequencies given by (20b) and (21a) and with the inverse values of the lifetime of the Mössbauer nucleus in the excited state) relaxation of magnetizations of sublattices. This case is often implemented for ferromagnetic nanoparticles when the relaxation processes are determined by the amplitude of the random magnetic field induced by the dipole-dipole interaction between particles $[18,22,23$, 26]. For antiferromagnetic nanoparticles, this limiting case is even more justified from the physical point of view since their resulting magnetic moments (in low-lying excitation states) are much less than the uniform magnetization of ferromagnetic particles, and the characteristic precession frequencies of spins (20b) and (21a) are several orders of magnitude higher.

In this case, the equilibrium state of the ensemble of particles at a given temperature $T$ is described by the Gibbs distribution over the "quasistationary" states (precession and nutation trajectories of the vectors $\mathbf{M}_{1}$ and $\mathbf{M}_{2}$ ) with given $E$ and $m$ values, each of which is characterized by the average values of the longitudinal components of magnetizations of sublattices $\bar{m}_{1 z}(E, m)$ and $\bar{m}_{2 z}(E, m)=m-\bar{m}_{1 z}(E, m)$

$$
W(E, m)=C e^{-E V / k_{B} T}
$$

Here, $V$ is the volume of particles and $C$ is the constant given by the normalization condition

$$
2 \int d E W(E, m)\left[m_{\max }(E)-m_{\min }(E)\right]=1
$$

3.1. Mössbauer Spectra in the Absence of Hyperfine Quadrupolar Interaction. In our case, calculations of the Mössbauer spectra of the ensemble of chaotically oriented antiferromagnetic particles can be performed in accordance with the results obtained in $[18,22,23]$ taking into account nonequivalence of the vectors $\mathbf{M}_{1}$ and $\mathbf{M}_{2}$ in the presence of uncompensated magnetic moment with the excitation spectra shown in Figures 2 and 4. In particular, the cross section for the absorption of a $\gamma$-ray photon with the energy $E_{\gamma}=\hbar \omega$ by antiferromagnetic particles with a given $\beta$ value is determined in the simplest case of the absence of the hyperfine quadrupolar interaction by the following expression:

$$
\begin{aligned}
& \sigma(\omega, \beta)=\frac{\sigma_{a}}{2} \int d E W(E) \int_{m_{\min }(E)}^{m_{\max }(E)} d m \\
& \cdot \sum_{i=1}^{2} \sum_{j}\left[L\left(\omega, \bar{m}_{i z}^{(j)}(E, m)\right)+L\left(\omega, \bar{m}_{i z}^{(j)}(E,-m)\right)\right] .
\end{aligned}
$$

Here, $\sigma_{a}$ is the effective absorber thickness and the partial absorption spectra corresponding to the "quasistationary" states are written in the standard form $[18,22,23]$ :

$$
L(\omega, x)=\frac{\Gamma_{0}^{2}}{4} \sum_{\alpha} \frac{\left|C_{\alpha}\right|^{2}}{\left(\widetilde{\omega}-x \omega_{\alpha}\right)^{2}+\Gamma_{0}^{2} / 4} .
$$


Here, $\Gamma_{0} \equiv \Gamma_{0} / \hbar$ is the width of the energy level of the excited nuclear state in frequency units; $\alpha=\left(m_{e}, m_{q}\right)$ specifies the hyperfine transitions with the projections of the nuclear spin on the direction of the hyperfine field for the ground, $m_{g}$, and excited, $m_{e}$, nuclear states; the Clebsch-Gordan coefficients $C_{\alpha}$ determine the intensities of transitions at the resonance frequencies

$$
\omega_{\alpha}=\omega_{e} m_{e}-\omega_{g} m_{g}
$$

where

$$
\omega_{g, e}=-\frac{g_{g, e} \mu_{N} H_{\mathrm{hf}}}{\hbar}
$$

are the Larmor frequencies of the precession of nuclear spin in the ground and excited states with the nuclear $g$-factors $g_{g}$ and $g_{e}$, respectively, $\mu_{N}$ is the nuclear magneton, and $\widetilde{\omega}=$ $\omega-E_{0} / \hbar$, where $E_{0}$ is the energy of the resonance transition. According to (49)-(52), the calculation of the absorption spectrum is reduced to averaging over the distribution of the effective hyperfine field for all trajectories of motion of magnetizations of sublattices given by the values $\bar{m}_{1 z}^{(j)}(E, m)$ and $\bar{m}_{2 z}^{(j)}(E, m)$ with weight factors (48a) and (48b), which are defined by the excitation spectrum of antiferromagnetic particles (Figures 2 and 4).

To calculate the absorption spectrum of the Néel ensemble of antiferromagnetic particles, one must also perform averaging over a random quantity of the uncompensated magnetic moment, for example, according to the Gaussian distribution of parameter $\beta$ with average magnitude $\bar{\beta}=1$ and width $\sigma_{\beta}$ :

$$
\bar{\sigma}(\omega)=\frac{2}{\sigma_{\beta} \sqrt{2 \pi}} \int_{0}^{1} \sigma(\omega, \beta) \exp \left(-\frac{(\beta-\bar{\beta})^{2}}{2 \sigma_{\beta}^{2}}\right) d \beta .
$$

Typical absorption spectra for an ensemble of slowly relaxing ideal $(\beta=1)$ and Néel $\left(\bar{\beta}=1, \sigma_{\beta}=0.03\right)$ antiferromagnetic particles calculated by (48a)-(52) are shown in Figure 5 . These calculations reflect the qualitative features of the energy spectrum of antiferromagnetic particles given in Section 2 and their manifestation in absorption spectra $[18,22]$, namely, the transition from the well-resolved magnetic hyperfine structure at low temperatures to the higher-temperature single line against the background of the magnetic hyperfine structure with sharply asymmetric lines at intermediate temperatures. Moreover, the performed generalization of the continuum model [18] to the case of the continuous nutation spectrum accompanying the normal modes of the selfconsistent precession of magnetizations of sublattices [22] makes it possible to completely fit quantitatively the results of the performed calculations with earlier quantum-mechanical calculations in the macroscopic limit $\left(k S^{2} \gg 1\right)[7,8]$, including the high-temperature collapse of the magnetic hyperfine structure into the single line (or the quadrupolar doublet of lines). The latter allows one principally to treat a lot of experimental Mössbauer spectra earlier collected on antiferromagnetic nanoparticles [9-12] just as it has been made in [17] for ideal antiferromagnetic particles.

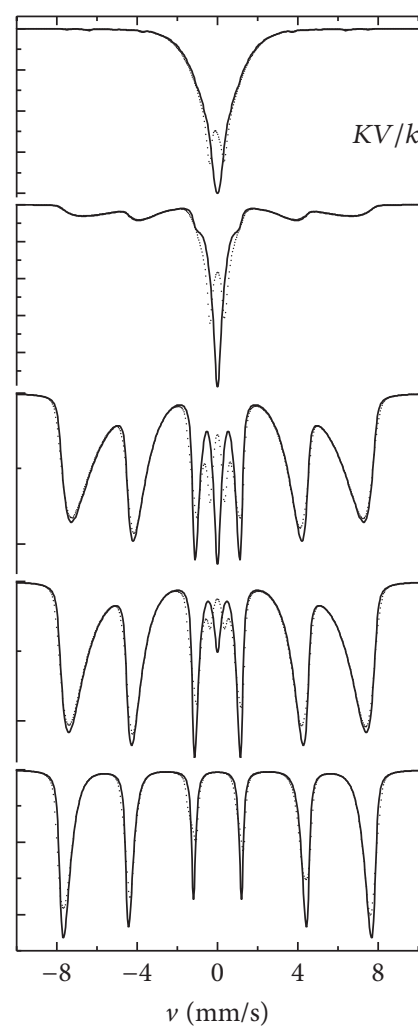

(a)

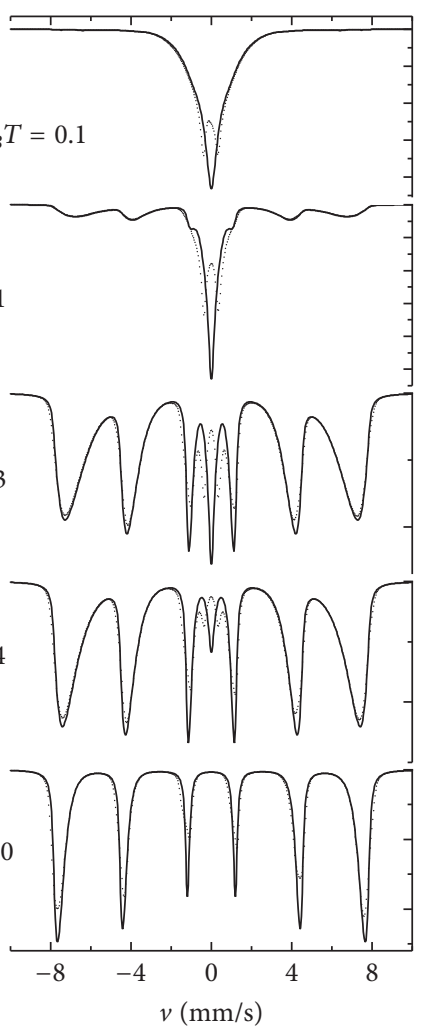

(b)
FIGURE 5: Mössbauer absorption spectra of ${ }^{57} \mathrm{Fe}$ nuclei in the ensemble of slowly relaxing ideal $(\beta=1)$ antiferromagnetic particles (a) and the Néel ensemble of slowly relaxing "uncompensated" $(\bar{\beta}=$ $1, \sigma_{\beta}=0.03$ ) antiferromagnetic particles (b) calculated within the model of magnetic dynamics (see main text) for different values of the effective energy barrier $K V / k_{B} T$ in the presence (dotted lines) of the quadrupolar interaction with the constant $q=0.35 \mathrm{~mm} / \mathrm{s}$ and in the absence (solid lines) of this interaction $(q=0)$. Hereinafter, $H_{\mathrm{hf}}=500 \mathrm{kOe}$.

These calculations confirm also the fundamental conclusion following the quantum model that the uncompensated spin (magnetic moment) in the limit of the slow magnetization relaxation does not alter the qualitative character of the shape evolution for the spectra of ideal antiferromagnetic particles with temperature [14]; rather, it leads just to slight quantitative corrections in the central part of the spectrum that are seen only at high temperatures. The detailed character of such corrections is demonstrated in Figure 6, where partial absorption spectra corresponding to nonequivalent magnetic sublattices with magnetizations $\mathbf{M}_{1}$ and $\mathbf{M}_{2}$ are shown for different values of parameter $\beta$.

3.2. Mössbauer Spectra in the Presence of Hyperfine Quadrupolar Interaction. Equations (49)-(51b) are valid only in the absence of the hyperfine quadrupolar interaction of the nuclear magnetic moment with the atomic electron shell. As long as in most cases the experimental Mössbauer spectra of magnetic nanoparticles display the high-temperature collapse of the magnetic hyperfine structure into a quadrupolar 


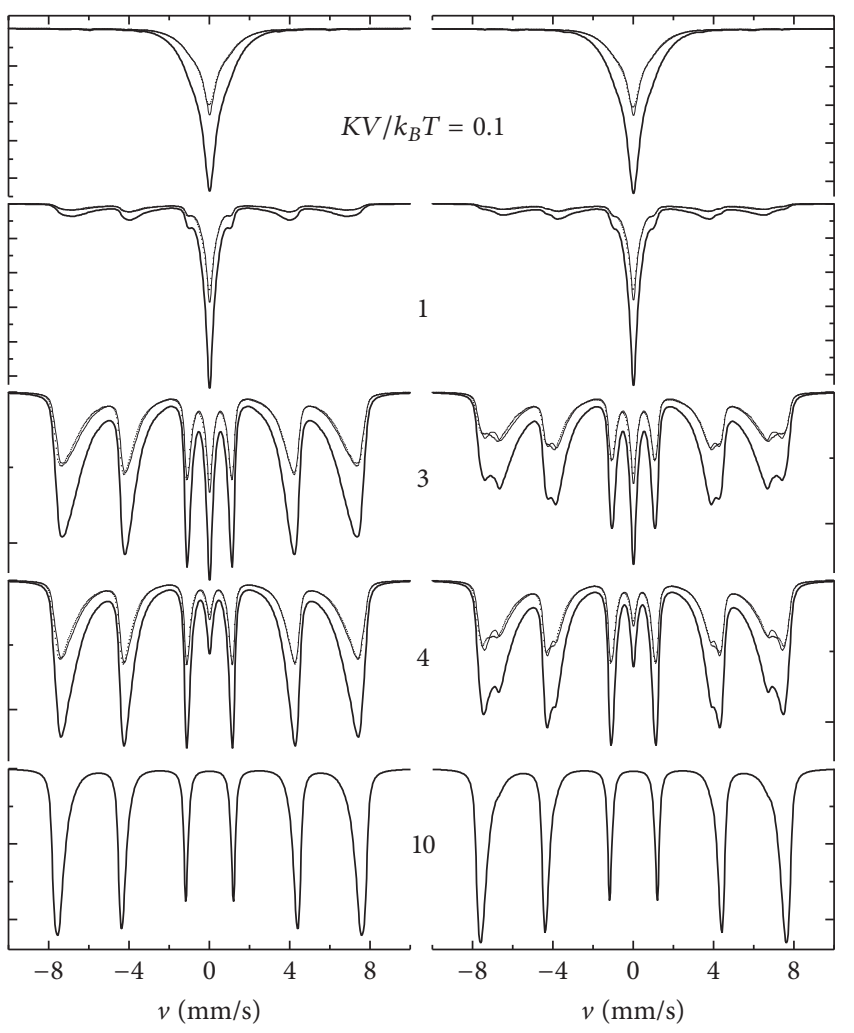

(a)

(b)

FIgURE 6: Mössbauer absorption spectra (solid lines) of ${ }^{57} \mathrm{Fe}$ nuclei in the ensemble of "uncompensated" antiferromagnetic particles with $\beta=0.99$ (a) and $\beta=0.97$ (b) calculated in the slow-relaxation regime for different values of the effective energy barrier $K V / k_{B} T$ in the absence of the quadrupolar interaction $(q=0)$. Partial absorption spectra corresponding to the sublattice magnetizations $\mathbf{M}_{1}$ (thin solid lines) and $\mathbf{M}_{2}$ (dotted lines) are also shown.

doublet of lines [9-13, 23], but not into a single line, one should perform generalization of a formalism described in the previous section on the presence of the hyperfine quadrupolar interaction. To solve the problem one can use the results obtained in [26], where the cross section for absorption of a gamma-quantum by an ensemble of magnetic nanoparticles is determined by the averaging over the chaotic distribution of the easy magnetization axes with respect to the principal axis $z^{\prime}$ of the electric field gradient at the nucleus, which in our case of the Néel ensemble of antiferromagnetic particles can be written as

$$
\sigma(\omega, \beta)=\int \sigma(\omega, \beta, \Theta) \sin \Theta d \Theta
$$

Here $\Theta$ is the angle between the axes $z$ and $z^{\prime}$.

Then, the general equations for calculations of partial absorption spectra $\sigma(\omega, \Theta)$ in [26] can be generalized for the presence of uncompensated magnetic moment in antiferromagnetic particles. By analogy with (49), the absorption spectrum of ${ }^{57} \mathrm{Fe}$ nuclei in the ensemble of antiferromagnetic particles in the presence of the hyperfine quadrupolar interaction and in the slow-relaxation regime can be represented in the following form:

$$
\begin{aligned}
& \sigma(\omega, \beta, \Theta)=\frac{\sigma_{a}}{2} \int d E W(E) \int_{m_{\min }(E)}^{m_{\max }(E)} d m \\
& \cdot \sum_{i=1}^{2} \sum_{j}\left[L\left(\omega, \bar{m}_{i z}^{(j)}(E, m), \Theta\right)\right. \\
& \left.+L\left(\omega, \bar{m}_{i z}^{(j)}(E,-m), \Theta\right)\right] .
\end{aligned}
$$

Here,

$$
\begin{aligned}
& L(\omega, x, \Theta)=-\frac{\Gamma_{0}}{6} \operatorname{Im} \sum_{\eta} \sum_{\substack{m_{g} m_{e} \\
j, \widetilde{m}_{j}}} V_{m_{g} \widetilde{m}_{j}}^{(\eta)+} \\
& \cdot \frac{\left\langle m_{e} \mid \widetilde{m}_{j}\right\rangle}{\widetilde{\omega}-\widetilde{\lambda}_{j}(x, \Theta)+x \omega_{g} m_{g}+i \Gamma_{0} / 2} V_{m_{e} m_{g}}^{(\eta)},
\end{aligned}
$$

$\tilde{\lambda}_{j}(\Theta, x)$ are the eigenvalues of the Hamiltonians of combined hyperfine magnetic and quadrupolar interactions for the excited nuclear state:

$$
\begin{aligned}
\widehat{H}^{(e)}(x, \Theta) & =\widehat{H}_{\mathrm{hf}}^{(e)}(x)+\widehat{H}_{\mathrm{Q}}^{(e)}(\Theta) \\
& =x \omega_{e} \widehat{I}_{z}^{(e)}+q\left[\widehat{I}_{z^{\prime}}^{2}-\frac{1}{3} I_{e}\left(I_{e}+1\right)\right\rfloor,
\end{aligned}
$$

where $I_{g}=1 / 2$ and $I_{e}=3 / 2$ are the nuclear spins, $\widehat{I}_{z}^{(e)}$ and $\widehat{I}_{z^{\prime}}$ are the operators of the projections of the nuclear spin in the excited state onto the easy axis and the direction of the principal axis $z^{\prime}$ of the electric field gradient at the nucleus, respectively, $q$ is the constant of quadrupolar interaction, $\widetilde{m}_{j} \equiv \widetilde{m}_{j}(x, \Theta)$ are the projections of the nuclear spin in the excited state onto the quantization axis for which the Hamiltonian (56) is diagonal, and $V_{m_{e} m_{g}}^{(\eta)}$ are the matrix elements of the operator of interaction of the gamma-quantum with a given polarization $\eta$ and the nucleus. The sum over random polarization $\eta$ in (54) for the chaotic distribution of the easy magnetization axes and unpolarized source of radiation is reduced to the sum over three orthogonal polarizations.

As seen from (53)-(56), the shape of the absorption spectrum for an ensemble of antiferromagnetic particles is defined both by the averaging over the distribution of the effective hyperfine field given by the values $\bar{m}_{1 z}^{(j)}(E, m)$ and $\bar{m}_{2 z}^{(j)}(E, m)$ with weight factors (48a) and (48b) and the ratios $q /\left(x \omega_{e}\right)$ in the Hamiltonian (56) [26]. Typical absorption spectra for an ensemble of slowly relaxing ideal $(\beta=1)$ and Néel $\left(\bar{\beta}=1, \sigma_{\beta}=0.03\right)$ antiferromagnetic particles in the presence of the hyperfine quadrupolar interaction, which was calculated by (53)-(56), are shown by dotted lines in Figure 5. These spectra demonstrate both the specific shapes of the hyperfine magnetic structure, reflecting qualitative features of the energy spectrum of antiferromagnetic particles, described in the previous section, and the transition from the well-resolved magnetic hyperfine structure at low temperatures to the higher-temperature quadrupolar doublet 
of lines, which is usually observed in the experiments [9$13,23]$. It is clearly seen that in this case also the presence of uncompensated magnetic moment in the limit of the slow magnetization relaxation does not alter the qualitative character of the shape evolution for the spectra of ideal antiferromagnetic particles with temperature [14] but modifies slightly the central part of the spectrum calculated at high temperatures. These corrections are even smaller than those observed in the absence of the hyperfine quadrupolar interaction because of existence of additional averaging (53).

\section{Ferrimagnetic Nanoparticles}

The normal precession modes of vectors $\mathbf{M}_{1}$ and $\mathbf{M}_{2}$, the excitation branches in the energy spectrum, and ranges of the allowed characteristics of nutations of the sublattice magnetizations of antiferromagnetic particles with $\beta=0.5$ (lower panels in Figures 2 and 4) formally correspond to ferrimagnetic particles. These normal modes correspond to the classical theory of ferromagnetic resonance in the presence of two sublattices [24], for which two types of the precession of sublattice magnetizations are characteristic: low-frequency and high-frequency. Normal mode 1 in Figure 2 corresponds to the low-frequency precession of oppositely directed vectors $\mathbf{M}_{1}$ and $\mathbf{M}_{2}$ while normal modes 2 and 3 correspond to the high-frequency precession of these vectors at different angles to the axis of anisotropy. For modes 2 and 3 the orientation of vector $\mathbf{M}_{1}$ with a smaller magnetic moment is varied over the entire range of possible values of polar angle $\theta$, while vector $\mathbf{M}_{2}$ is reoriented in the strictly limited range of $\theta$ :

$$
\begin{aligned}
& m_{2 z}^{(2)} \geq \sqrt{1-\beta^{2}}, \\
& m_{2 z}^{(3)} \leq-\sqrt{1-\beta^{2}},
\end{aligned}
$$

whereas the deviation of vector $\mathbf{M}_{2}$ from the easy axis is always much less than the one for vector $\mathbf{M}_{1}$ (Figure 2).

Using the mathematical formalism described in the previous sections, one can calculate also Mössbauer absorption spectra of an ensemble of antiferromagnetic particles with uncompensated magnetic moment for arbitrary values of parameter $\beta$, in particular, for ferrimagnetic particles. Examples of such calculations for $\beta=0.5$ and slow magnetic relaxation regime are shown in Figure 7. These calculations reflect features of the orientation dependence of the normal precession modes and nutations of the magnetization vectors of sublattices and the excitation energy spectrum of particles with $\beta=0.5$ (Figures 2 and 4). The difference between the shapes of partial spectra for two magnetic sublattices grows monotonically with an increase in temperature (Figure 7). At high temperatures, the specific shape of the resulting spectrum and both partial spectra is observed in the form of a superposition of a smoothed magnetic hyperfine structure with lines of almost triangular shape and a broaden single line (or quadrupolar doublet not shown in Figure 7). Spectra of precisely this shape have often been observed in experiments [23].

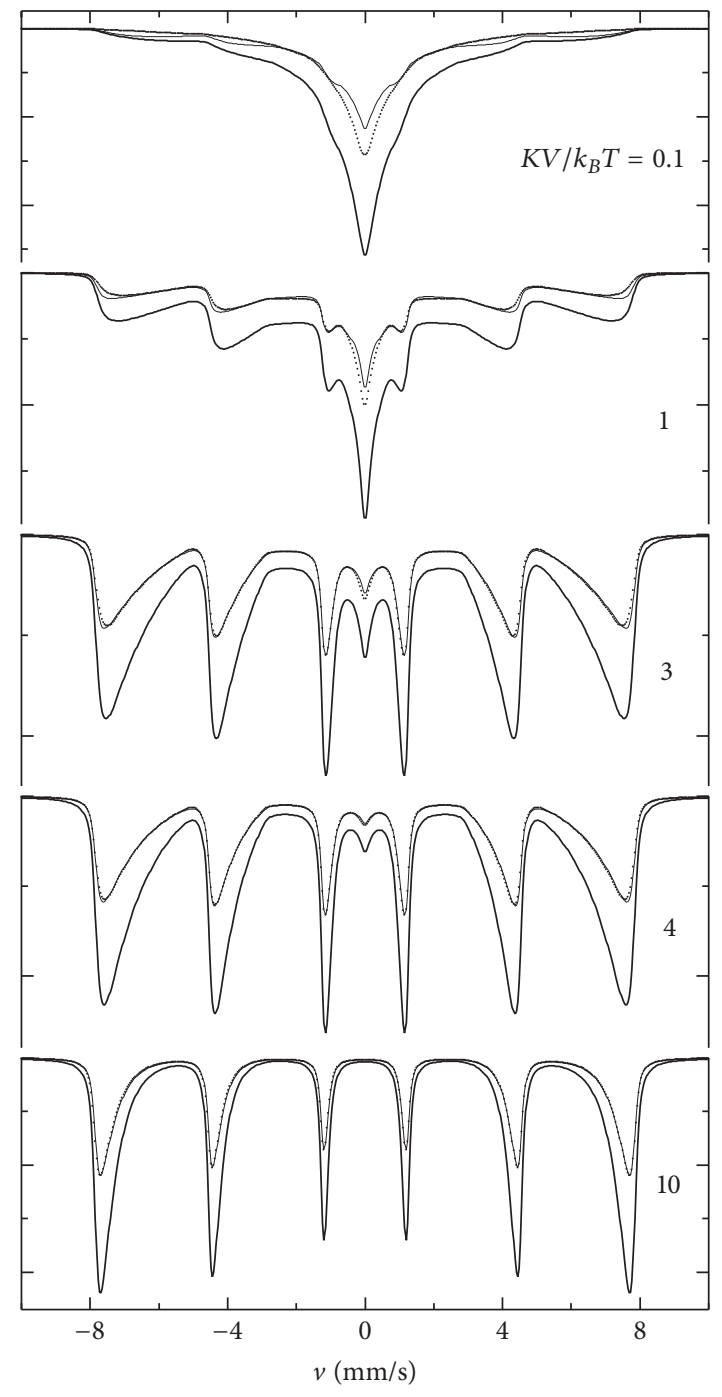

FIGURE 7: Mössbauer absorption spectra (solid lines) of ${ }^{57} \mathrm{Fe}$ nuclei in the ensemble of ferrimagnetic particles with $\beta=0.5$ calculated in the slow-relaxation regime for different values of the effective energy barrier $K V / k_{B} T$ in the absence of the quadrupolar interaction $(q=0)$. Partial absorption spectra corresponding to the sublattice magnetizations $\mathbf{M}_{1}$ (thin solid lines) and $\mathbf{M}_{2}$ (dotted lines) are also shown.

The normal precession modes and nutations of the magnetization vectors of sublattices as well as the excitation energy spectrum and absorption spectra of particles with $\beta=0.5$ (Figures 2 and 4 ) should be considered as only one possible limiting case for ferrimagnetic particles. To solve the problem in a general form, one must generalize the results from points of view on both magnetism (e.g., different values of the magnetic anisotropy constants for two sublattices in (1)) and Mössbauer spectroscopy (different values of hyperfine field and isomer shifts for two sublattices). General consideration of the problem lies beyond the scope of this work, but we note that the most serious problem one faces this way is classifying all possible forms of the manifestation of magnetism in ferrimagnetic nanoparticles. 
For example, the phenomenological theory of ferrimagnetism of bulk materials remains still based on the Néel classification according to the temperature dependence of saturation magnetization and describes seven main types of such dependence at once [27]. At the same time, an adequate phenomenological theory for describing magnetism of ferrimagnetic nanoparticles is actually absent so that the question of qualification of the materials has even not been raised. In this aspect besides the results given in this section it is possible to call only the four-level relaxation model in two-sublattice approximation for describing the Mössbauer spectra of ferrimagnetic nanoparticles under conditions of metamagnetism [25]. Note that the experimental absorption spectra of ferrimagnetic nanoparticles display a number of shapes, which are qualitatively different from those for bulk materials; in particular, they manifest particles size effects in a clear form (see, e.g., Fig. 1 in [25]).

\section{Conclusions}

Thus, a theory for describing excitation spectrum and thermodynamic properties of the Néel ensemble of antiferromagnetic nanoparticles with uniaxial magnetic anisotropy and uncompensated magnetic moment is developed by means of generalization of the continual model of magnetic dynamics of ideal antiferromagnetic nanoparticles in the two-sublattice approximation. This approach clarifies the principal difference in thermodynamic behavior of ferromagnetic and antiferromagnetic particles revealed in spectroscopic measurements. In the framework of the theory, a formalism for calculating Mössbauer absorption spectra of an ensemble of antiferromagnetic particles is implemented, which can be efficiently used for analyzing a large array of experimental spectra taken on these materials so far. The resulting model can be easily realized on PC, which makes it possible to take into account the physical mechanisms of the formation of the hyperfine structure of the absorption spectra in the real situation and to numerically describe the qualitative features of the temperature evolution of the spectral shape, which is observed in most experimental spectra of ${ }^{57} \mathrm{Fe}$ nuclei in antiferromagnetic nanoparticles.

Calculations performed within the macroscopic theory confirm completely the main conclusions of the earlier developed quantum-mechanical model $[7,8,14]$ for an ensemble of antiferromagnetic particles. In particular, a nontrivial form of the excitation spectrum of such particles in the form of four excitation branches corresponding to the normal modes of self-consistent regular precession of magnetizations of sublattices and the continuous spectrum of nutations of magnetizations accompanying these normal modes allows one to qualitatively describe both the characteristic shapes of the Mössbauer spectra depending on temperature and to give a phenomenological interpretation of macroscopic quantum effects observed earlier in experimental absorption spectra $[9-13,17,23]$ and described within the quantummechanical representation $[7,8,14]$. Consecutive use of both the models, macroscopic and quantum-mechanical, in analyzing experimental data will allow one to establish reliably existence and to classify the macroscopic quantum effects defining shapes of experimental absorption spectra. However, full-scale comparison of two models demands to generalize them for the presence of continuous magnetic relaxation processes; the present macroscopic model is an excellent basis for the solution of such a problem.

It is worth mentioning that the above model of magnetic dynamics and the formalism for calculations of the Mössbauer spectra of an ensemble of antiferromagnetic particles can be easily generalized to the case of antiferromagnetic and ferrimagnetic nanoparticles in an external magnetic field. Solution of the problem in the last case will allow a more accurate description of not only the Mössbauer spectra in a magnetic field [28] but also the magnetization curves of an ensemble of nanoparticles [29] taking directly into account the magnetic nature inherent to the particles.

\section{Conflicts of Interest}

The author declares that there are no conflicts of interest regarding the publication of this paper.

\section{Acknowledgments}

The author is grateful to the Russian Foundation for Basic Research for a financial support.

\section{References}

[1] L. Néel, "Superparamagnétisme des grains très fins ferromagnétiques," Comptes Rendus de l'Académie des Sciences. Paris, vol. 252, p. 4075, 1961.

[2] L. Néel, "Superposition de l'antiferromagnétisme et du superparamagnétisme dans un grain très fin," Comptes Rendus de l'Académie des Sciences. Paris, vol. 253, p. 9, 1961.

[3] C. Gilles, P. Bonville, K. K. W. Wong, and S. Mann, "NonLangevin behaviour of the uncompensated magnetization in nanoparticles of artificial ferritin," The European Physical Journal B, vol. 17, pp. 417-427, 2000.

[4] N. J. O. Silva, A. Millán, F. Palacio et al., “Temperature dependence of antiferromagnetic susceptibility in ferritin," Physical Review B - Condensed Matter and Materials Physics, vol. 79, no. 10, Article ID 104405, 2009.

[5] R. H. Kodama and A. E. Berkowitz, "Atomic-scale magnetic modeling of oxide nanoparticles," Physical Review B, vol. 59, p. 6321, 1999.

[6] P.-A. Lingård, "Theory and simulation of antiferomagnetic nano-particles," Journal of Magnetism and Magnetic Materials, vol. 266, no. 1-2, pp. 88-95, 2003.

[7] M. A. Chuev, "On the thermodynamics of antiferromagnetic nanoparticles by example of Mössbauer spectroscopy," JETP Letters, vol. 95, no. 6, pp. 295-301, 2012.

[8] M. A. Chuev, "Macroscopic quantum effects observed in Mössbauer spectra of antiferromagnetic nanoparticles," Hyperfine Interactions, vol. 226, no. 1-3, pp. 111-122, 2014.

[9] W. Kündig, H. Bömmel, G. Constabaris, and R. H. Lindquist, "Some properties of supported small $\alpha$-Fe2O3 particles determined with the mössbauer effect," Physical Review, vol. 142, no. 2, pp. 327-333, 1966.

[10] I. P. Suzdalev, V. N. Buravtsev, V. K. Imshennik et al., "Magnetic properties of ultrafine ferrihydrite clusters studied by 
Mossbauer spectroscopy and by thermodynamical analysis," Zeitschrift für Physik D Atoms, Molecules and Clusters, vol. 37, pp. 55-61, 1996.

[11] F. Bødker, M. F. Hansen, C. B. Koch, K. Lefmann, and S. Mørup, "Magnetic properties of hematite nanoparticles," Physical Review B, vol. 61, p. 6826, 2000.

[12] I. P. Suzdalev, Y. V. Maksimov, V. K. Imshennik et al., "Magnetic phase transitions in nanostructures with different cluster orderings," Nanotechnologies in Russia, vol. 4, no. 7-8, pp. 467-474, 2009.

[13] L. Bordonali, Y. Furukawa, M. Kraken et al., " 1 H-NMR study of the spin dynamics of fine superparamagnetic nanoparticles," Physical Review B, vol. 85, no. 17, Article ID 174426, 2012.

[14] M. A. Chuev, "The role of an uncompensated spin in the formation of a hyperfine structure of the Mössbauer spectra of antiferromagnetic nanoparticles," Doklady Physics, vol. 57, no. 11, p. 421, 2012.

[15] J. M. Duan and A. Carg, "Macroscopic quantum coherence in small antiferromagnetic particles," Journal of Physics: Condensed Matter, vol. 7, p. 2171, 1995.

[16] Y.-H. Nie, Y.-B. Zhang, J.-Q. Liang, H. J. W. Müller-Kirsten, and F.-C. Pu, "Macroscopic quantum coherence in small antiferromagnetic particles and quantum interference effects," Physica B: Condensed Matter, vol. 270, no. 1-2, pp. 95-103, 1999.

[17] I. N. Mischenko and M. A. Chuev, "Quantum-mechanical and continual models of magnetic dynamics for antiferromagnetic particles in Mössbauer spectra analysis," Hyperfine Interactions, vol. 237, p. 21, 2016.

[18] M. A. Chuev, "Excitation spectrum and magnetic dynamics of antiferromagnetic nanoparticles in Mössbauer spectroscopy," JETP Letters, vol. 99, no. 5, pp. 278-282, 2014.

[19] C. Kittel, "Theory of antiferromagnetic resonance [29]," Physical Review, vol. 82, no. 4, p. 565, 1951.

[20] F. Kiffer and C. Kittel, "Theory of Antiferromagnetic resonance," APS Journals Archive, vol. 85, p. 329, 1952.

[21] L. D. Landau and E. M. Lifshitz, Mechanics, Pergamon, New York, NY, USA, 1988.

[22] M. A. Chuev, "Nutations of magnetizations of sublattices and their role in the formation of Mössbauer spectra of antiferromagnetic nanoparticles," JETP Letters, vol. 103, no. 3, pp. 175180, 2016.

[23] M. A. Chuev and J. Hesse, Magnetic Properties of Solids, Nova Science, New York, NY, USA, 2009.

[24] R. K. Wangsness, "Sublattice effects in magnetic resonance," Physical Review, vol. 91, no. 5, pp. 1085-1091, 1953.

[25] M. A. Chuev, "On the shape of gamma-resonance spectra of ferrimagnetic nanoparticles under conditions of metamagnetism," JETP Letters, vol. 98, no. 8, pp. 465-470, 2013.

[26] M. A. Chuev, "Multi-level relaxation model for describing the Mössbauer spectra of single-domain particles in the presence of quadrupolar hyperfine interaction," Journal of Physics: Condensed Matter, vol. 23, Article ID 426003, 2011.

[27] L. Néel, "Propriétés magnétiques des ferrites; ferrimagnétisme et antiferromagnétisme," Annales de physique (Paris), vol. 12, no. 3, p. 137, 1948.

[28] M. A. Chuev, "Multilevel relaxation model for describing the Mössbauer spectra of nanoparticles in a magnetic field," JETP, vol. 114, no. 4, p. 609, 2012.

[29] M. A. Chuev and J. Hesse, "Nanomagnetism: extension of the Stoner-Wohlfarth model within Néel's ideas and useful plots," Journal of Physics: Condensed Matter, vol. 19, Article ID 506201, 2007. 

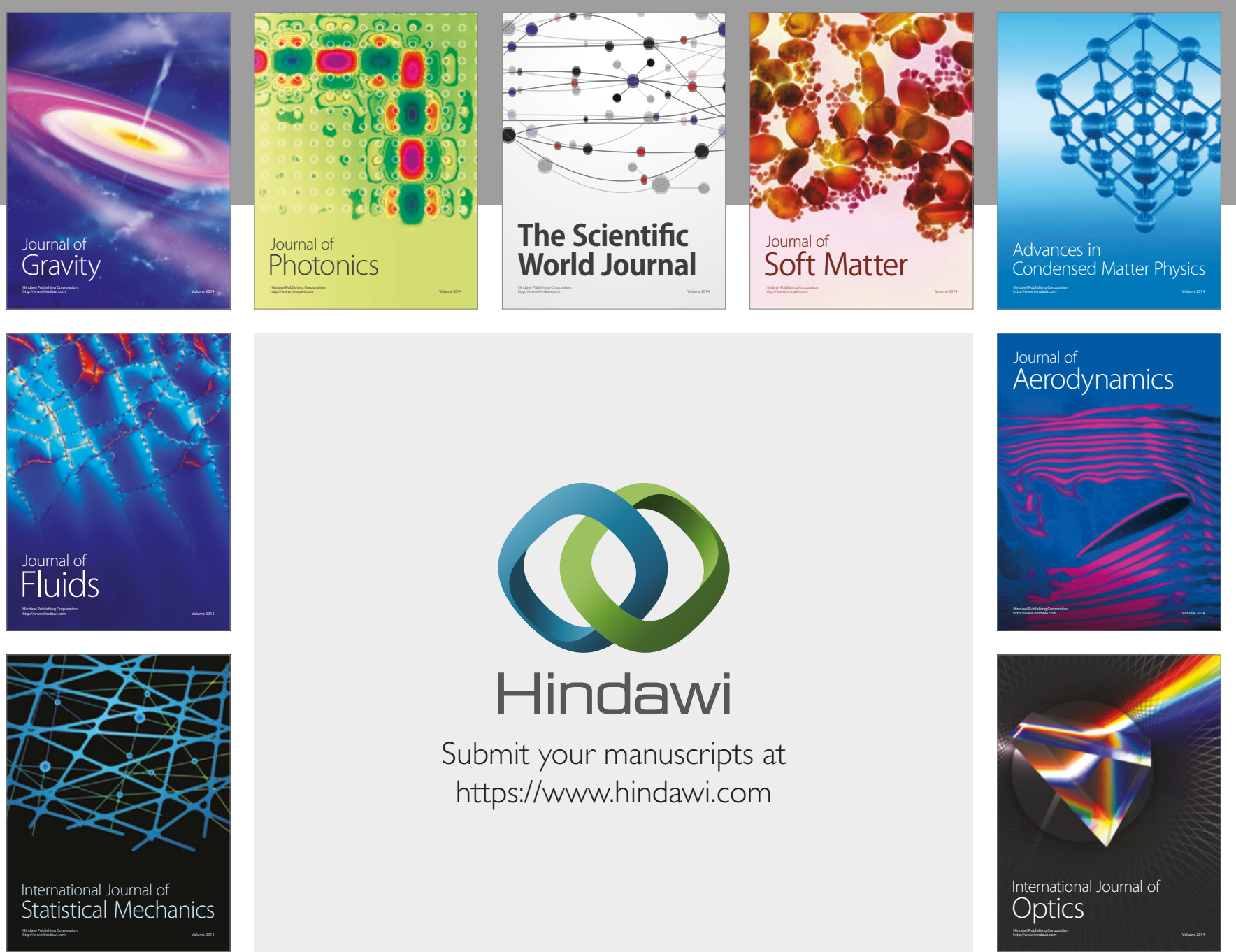

Submit your manuscripts at

https://www.hindawi.com
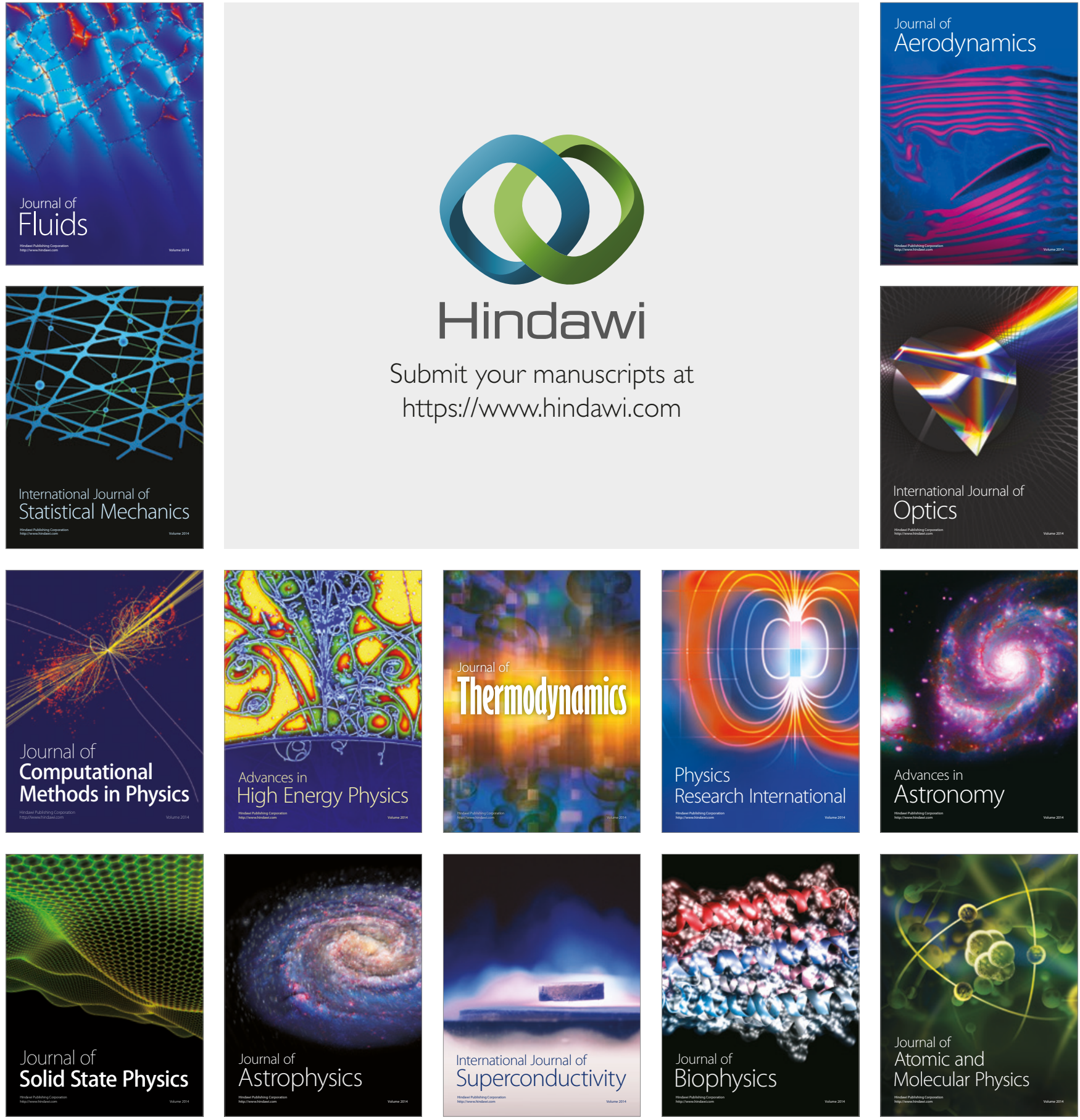